\title{
Association of Doppler Wave Pattern of Hepatic Veins and Fatty Liver Disease Degree
}

\section{Cibele F Carvalho ${ }^{1 *}$, Márcia M Jerico ${ }^{2}$, Bruno Cogliati ${ }^{3}$, Thassila C F Cintra ${ }^{4}$ and Maria Cristina Chammas ${ }^{5}$}

${ }^{1}$ Diplomate of the Brazilian College of Veterinary Radiology, Institute of Radiology, University of São Paulo, SP, Brazil

${ }^{2}$ Private Practice Endocrinology, São Paulo, SP, Brazil

${ }^{3}$ Department of Pathology of Veterinary Medicine School, University of São Paulo, SP, Brazil

${ }^{4}$ Private Practice Ultrasonography, São Paulo, SP, Brazil

${ }^{5}$ Diplomate of the Brazilian College of Radiology, Institute of Radiology, University of São Paulo, SP, Brazil

\begin{abstract}
The objective of this study was to document the progressive effects of fatty infiltration of the liver on hepatic veins (HVs) by measuring Doppler waveforms in groups of rats and dogs. All of the groups underwent hepatic B-mode and duplex Doppler sonography. B-mode fatty infiltration was classified into four degrees corresponding to increasing grades of hepatic echogenicity for all groups: (0) absent, (1) mild, (2) moderate and (3) severe fatty infiltration. Histograms were obtained in all dogs to standardise each grade of hepatic echogenicity and an increasing distribution of grey-levels was found according to the grade of fatty infiltration of the liver in obese dogs. The Doppler sonography spectra of HVs were classified into three groups: normal or triphasic waveforms, biphasic waveforms, and monophasic or flat waveforms. Obese dogs $(60 \%)$ and rats $(100 \%)$ with fatty infiltration of the liver presented biphasic or flat right HV Doppler waveforms more often than control dogs and rats, and these differences were statistically significant ( $p=0.002$ for dogs and $p=0.0028$ for rats). None of the control dogs and rats had monophasic waveforms. These findings suggest there is an association between wave form pattern of hepatic veins and the degree of fatty liver disease.
\end{abstract}

Keywords: Hepatic disease; Obesity; Ultrasonography; Small animal; Dogs; Rats; Duplex doppler

\section{Introduction}

Obesity is defined as the accumulation of excessive amounts of adipose tissue in the body [1]. Most investigators agree that, as in humans, the incidence of obesity in the pet population is increasing, and one study estimated the incidence of obesity in the dog population to be $22-40 \%$ [2]

Fatty liver infiltration (steatosis or fatty liver disease) occurs in obese cats and can exist as a primary disorder or be experimentally induced in cats and dogs [3-5]. Hepatic lipidosis, steroid hepatopathies, and vacuolar hepatopathies are often present with other primary disorders in dogs or experimentally induced [6-9]. These hepatopathies are usually associated with a diffuse increase in parenchymal echogenicity and hepatomegaly [10].

Currently several noninvasive techniques can assess fatty infiltration of the liver, each with its own advantages and disadvantages. Computed tomography can detect hepatic fat content, but it involves ionizing radiation. Magnetic resonance spectroscopy is probably the most accurate and fastest method of detecting fat, but it is expensive and the necessary software is still not easily available in most units [11]. Maybe ultrasound is still in the first option for diagnosis, but its accuracy depends on the operator and the patient's features.

Doppler ultrasonography has become an important diagnostic method in the evaluation of hepatic vasculature and some hepatic parenchymal diseases [12]. The hepatic veins (HVs) drain blood from the hepatic sinusoids toward the caudal vena cava [13-15]. Normal HV Doppler waveforms have been widely reported in the literature, and the normal triphasic Doppler waveform is determined by the right atrial pressure, hepatic parenchymal compliance, and thoracic-abdominal pressure [16]. The flow pattern may be modified during the respiratory cycle, and some diseases impair HV compliance, thus reducing and flattening phasic oscillations $[16,17]$. HV flow is pulsatile, reflecting changes in the blood flow through the right heart chambers during the cardiac cycle $[16,18,19]$.

Recent reports in human medicine have suggested that fatty infiltration of the liver can cause changes in HV Doppler waveforms that could return to normal after weight loss treatment in some cases [17]. Accumulation of fatty deposits in the cytoplasm of hepatocytes is associated with an increase in cell volume, which reduces the size of the hepatic sinusoid space. As a result of impaired hepatic microcirculation, one study reported that ischaemia induces hepatic fibrogenesis in humans and rats, which may progress to liver cirrhosis if it persists over a long period [20]. Some liver injuries might induce fibrosis also in dogs, leading up to the development of portal hypertension [21]. To our knowledge, no prior studies have focused on the effects of fatty infiltration of the liver on HV Doppler waveforms in dogs. Using an experimental model of hepatosteatosis in rats, this work aimed to study the progressive effect of this disease on HV Doppler waveforms and compare these findings with clinical cases of obese dogs with diffuse fatty liver in the absence of any other diseases. It was also investigated whether HV Doppler waveforms might serve as a new method to assess the stages or degrees of fatty infiltration of the liver. That could be less complex or less invasive than existing methods such as grey scale analysis or histology of liver biopsies.

*Corresponding author: Carvalho CF, Travessa Leon Berry, 116. 01402-030 São Paulo, SP, Brazil, Tel: 55113884 2168; E-mail: cibelefcarvalho@gmail.com

Received November 07, 2014; Accepted January 15, 2015; Published January 19,2015

Citation: Carvalho CF, Jerico MM, Cogliati B, Cintra TCF, Chammas MC (2015) Association of Doppler Wave Pattern of Hepatic Veins and Fatty Liver Disease Degree. J Liver 4: 174. doi:10.4172/2167-0889.1000174

Copyright: (c) 2015 Carvalho CF, et al. This is an open-access article distributed under the terms of the Creative Commons Attribution License, which permits unrestricted use, distribution, and reproduction in any medium, provided the original author and source are credited. 


\section{Materials and Methods}

All procedures for animal experimentation were performed in accordance with the Helsinki Declaration of 1975 (NIH Publication no. 85-23, revised 1996) and the Guidelines of Animal Experimentation from the University of Sao Paulo School of Medicine and Animal Research: Reporting in vivo Experiments (ARRIVE).

The clinical group of dogs was composed of client-owned animals, and all procedures were performed with a high standard of veterinary care. The clients signed informed client consent forms permitting the use of their animals' data.

The present study was reviewed and approved by The Ethics Committee of São Paulo University, process number 358/10.

\section{Experimental group (rats)}

Twenty healthy adult Sprague-Dawley rats weighing 250-300 g each were housed under controlled conditions $\left(22 \pm 2^{\circ} \mathrm{C} ; 65 \pm 15 \%\right.$ relative humidity; $12 \mathrm{~h}$ light/dark cycle). All animals received humane care in accordance with the guidelines of the Committee on Care and Use of Animal Resources of the School of Medicine of the University of São Paulo. Ten rats served as the control group. Fatty infiltration of the liver was induced in 10 rats through a choline-deficient, highfat diet (35\% total fat, $54 \%$ trans fatty acid enriched) for 16 weeks (Rhoster Ltda, Brazil). The remaining rats served as a control group. All animals underwent B-mode and Doppler ultrasonography with a $12 \mathrm{MHz}$ linear transducer (Aplio XG, Toshiba Corp., Tokyo, Japan) under anaesthesia $(0.2 \mathrm{mg} / \mathrm{kg}$ ketamine hydrochloride $)$ weekly. To monitor the development of fatty liver disease, one rat was euthanased at week 4 and another at week 10 with overdoses of anaesthesia (ketamine hydrochloride). After euthanasia, all the rat livers were assessed grossly and by microscopy. Samples were fixed in $4 \%$ formaldehyde and processed for haematoxylin-eosin and Sirius red staining for histological analyses. The variables were scored by a blinded experienced hepatopathologist using a modified scoring system assessing macro/microsteatosis (0-3), hepatocyte ballooning (0-2), lobular inflammatory changes (0-3) and fibrosis (0-4) [22].

\section{Clinical group (dogs)}

Fifteen obese dogs (body score $>4 / 5$ ) with no other disease (mean age of 4.5 years; range: 2-6 years of age) were selected by ultrasonography for hepatosteatosis (one Shetland Sheepdog, five mongrels, one Scottish terrier, three beagles, two English cocker spaniels, one schnauzer, and two pinschers) [1]. Ten healthy dogs (body score 3/5) without sonographic findings of fatty liver disease (mean age of 2.5 years; range: $2-4$ years) were enrolled as a control group (four beagles, two maltese, two English cocker spaniels, one Yorkshire terrier, and one standard poodle). For all dogs, endocrinopathies were excluded by adrenal and thyroid function tests, and chronic hepatic diseases were excluded by clinical examination and biochemical liver function tests of measurement of enzyme activity (alkaline phosphatase, $\gamma$-glutamyltransferase, alanine aminotransferase and aspartate aminotransferase). Cardiac diseases were excluded by thoracic radiography, electrocardiogram, echocardiography, and clinical examinations.

After accounting for the potential complications of liver biopsies in these animal, we did not perform biopsies on all subjects [23]. Liver biopsies were performed on six animals (two from each grade of fatty infiltration of the liver, as detected by ultrasonography) to confirm the sonographic pattern of classification. All of the owners signed procedure consent forms. Percutaneous biopsies of the liver were guided by ultrasound as described previously and performed with a 14 G Tru-cut needle (Bard Maxi Core disposable instrument, Bard, USA) [23]. The dog biopsy samples were handled the same as the rat liver necropsy samples.

\section{Ultrasonography}

In all groups, B-mode and Doppler ultrasonography were performed by a single expert using colour Doppler equipment (Aplio XG, Toshiba Corp., Tokyo, Japan). The sonographer was blind for the diet of the rats. The experimental group was scanned with a $12 \mathrm{MHz}$ linear probe. The healthy and clinical dog groups were scanned with a $5-7 \mathrm{MHz}$ micro convex probe with the focus at the gallbladder level. All animals were positioned in variable recumbency (dorsally and/or left lateral). The liver was carefully scanned, and vascular malformations and spaceoccupying lesions were excluded. Using B-mode ultrasonography, the presence or absence and the severity of fatty infiltration were graded on a scale from 0 to 3, which corresponded to increasing grades of hepatic echogenicity (right lobes compared with echogenicity of the right kidney cortex and quadrate and left lobes compared with falciform fat) and attenuation of the sound beam in the distal field (with poor visualisation of the intrahepatic vessels and diaphragm), to indicate, respectively: normal liver (0) and mild (1), moderate (2) or severe (3) fatty infiltration of the liver. Grey-level histograms were obtained using the program Photoshop CS4 (Adobe Systems Incorporated, San Jose, California, United States) to standardise each grade of hepatic echogenicity compared to falciform fat using the same level, imaging plane, and machine settings in both groups (healthy and clinical dogs). Digital images were transferred to a computer for a second line analysis. The sonographer was blind for the ultrasonic classification of hepatic steatosis in the dogs also. Histograms provided measures referring to the echogenicity (LMEAN), echotexture (NMOST/NALL) and echo amplitude standard deviation (SD).

The hepatic and portal anatomies of the rats and dogs were evaluated as described previously [13,24]. After the right HV was depicted intercostally along its longitudinal axis with colour Doppler flow mapping, pulsed Doppler was obtained in the right $\mathrm{HV}$ at a distance of $5 \mathrm{~mm}$ from the junction of the caudal vena cava, with a gate of $2 \mathrm{~mm}$ in the experimental group (rats) and a distance of $2 \mathrm{~cm}$ from that point in both groups of dogs (healthy and clinical groups), with a gate of 2 or $3 \mathrm{~mm}$. Special attention was addressed to obtained a correct insonation angle (set to less than $60^{\circ}$ ) to maximize Doppler signal. Doppler waveforms of the right HV were recorded when three consecutive waves were obtained. The normal triphasic HV Doppler waveform consists of two antegrade flow peaks during atrial and ventricular diastole, followed by a short interval of reversed flow peak during atrial contraction [16]. To standardise the technique, we chose the right HV because this vein is nearest to the caudal vena cava. The HV Doppler waveforms were classified as triphasic (normal with reversed flow in at least one phase), biphasic (no reversed flow and with or without decreased phasic oscillation) or monophasic (flat or complete loss of normal oscillation), as illustrated in Figure 1.

Data were expressed as medians and percentages. All $P$ values were two-tailed, and $\mathrm{P}<0.05$ was considered statistically significant. A chisquare test was used for analyses of categorical variables. Spearman's rank correlation was used to determine the relationship between fatty infiltration of the liver and the presence of abnormal HV Doppler waveforms. The sensitivity and specificity were calculated with Fisher's exact test. 


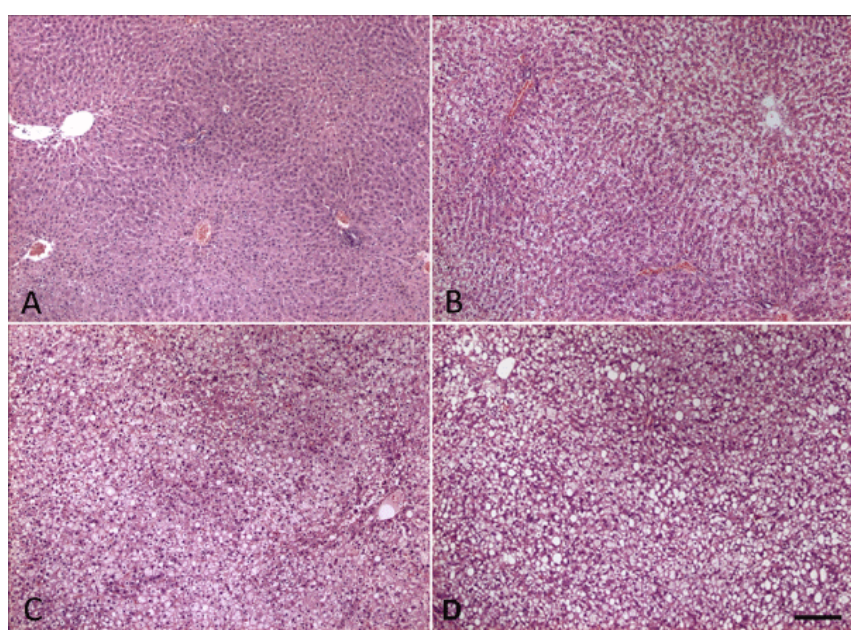

Figure 1: (A) Histology of normal liver parenchymal architecture of the control group (rat). (B) Liver histopathology of an obese rodent model with mild hepatocyte steatosis. (C) Liver histopathology of an obese rodent model with moderate hepatocyte steatosis. (D) Liver histopathology of an obese rodent model with severe hepatocyte steatosis (bar=50 $\mu \mathrm{m})$.

\section{Results}

Normal rats (control group) presented at various time points triphasic HV waveforms in $80-100 \%$ of subjects and biphasic HV waveforms in $0-20 \%$ of subjects. No monophasic waveform was found in the control group. An increasing distribution of abnormal HV flow patterns was not observed in the control group of rats over time (Table 1), but was observed in the rats fed the experimental diet according to the progression of fatty infiltration of the liver (Figure 1 and Table 2).

At the end of the study, the right $\mathrm{HV}$ waveforms were biphasic in $25 \%$ and monophasic in $75 \%$ of the experimental group of rats fed the diet to induce fatty infiltration of the liver. No triphasic waveform was found in this group.

In the experimental group (rats) it was also found an increasing echogenicity and grade of fatty infiltration of the liver at macro and microscopic steatosis results (Table 4). A statistical difference in the $\mathrm{HV}$ waveform flow was found between experimental and control rats $(\mathrm{P}=0.0028)$.

There was no statistical difference in the HV waveform flow for all obese dogs $(\mathrm{P}=0.22)$. For dogs that were given grades of 2 and 3 (moderate and severe fatty infiltration of the liver, respectively), there was a statistical difference in the severity of microscopic hepatocyte steatosis $(\mathrm{P}=0.002)$. Histograms were analysed to confirm the sonographic classification (Figure 2), and an increasing distribution of histogram grey-levels was found according to the grade of fatty infiltration of the liver in obese dogs (Table 3).

At the end of the study, both two clinical groups (moderate and severe steatosis) and the experimental group of rats presented an inverse relation between the sonographic grade of fatty infiltration of the liver and the phase of hepatic venous flow. Using waveforms as grades and the same system considered for histopathology, a triphasic waveform could be considered as grade 0 (normal or best), and a monophasic waveform could be considered as grade 3 (worst).

Normal dogs (control group) presented triphasic HV waveforms in $70 \%$ of animals and biphasic HV waveforms in 30\%. No monophasic waveform was found in the control group (Table 5). An increasing distribution of abnormal HV flow patterns was found according to the grade of fatty infiltration of the liver in obese dogs (Table 5).

Out of 15 obese dogs with steatosis, the Doppler flow pattern in the right $\mathrm{HV}$ was triphasic in six (40\%), biphasic in five (34\%), and monophasic in four (26\%) (Figure 3).

\section{Discussion}

The ability to accurately and noninvasively quantify fatty infiltration in organs such as the liver remains a critical component in understanding the link between obesity and its comorbidities such as steroid hepatopathies and fatty liver disease. Single-voxel $\left({ }^{1} \mathrm{H}\right)$ proton magnetic resonance spectroscopy has been regarded as

\begin{tabular}{|c|c|c|c|c|c|c|c|c|c|}
\hline Time (weeks) & $\mathbf{0}$ & $\mathbf{2}$ & $\mathbf{4}$ & $\mathbf{6}$ & $\mathbf{8}$ & $\mathbf{1 0}$ & $\mathbf{1 2}$ & $\mathbf{1 4}$ & $\mathbf{1 6}$ \\
\hline (n) & & & & & & & & & \\
\hline Triphasic & 10 & 8 & 9 & 10 & 9 & 10 & 10 & 8 & 9 \\
\hline Biphasic & - & 2 & 1 & - & 1 & - & - & 2 & 1 \\
\hline Monophasic & - & - & - & - & - & - & - & - & - \\
\hline
\end{tabular}

Table 1: Distribution of the HV flow pattern in control group of rats (normal).

\begin{tabular}{|c|c|c|c|c|c|c|c|c|c|}
\hline Time (weeks) & $\mathbf{0}$ & $\mathbf{2}$ & $\mathbf{4}$ & $\mathbf{6}$ & $\mathbf{8}$ & $\mathbf{1 0}$ & $\mathbf{1 2}$ & $\mathbf{1 4}$ & $\mathbf{1 6}$ \\
\hline $\mathbf{( n )}$ & & & & & & & & & \\
\hline Triphasic & 10 & 9 & 5 & 3 & 2 & - & - & - & - \\
\hline Biphasic & - & 1 & 4 & 5 & 6 & 7 & 5 & 4 & 2 \\
\hline Monophasic & - & - & - & 1 & 1 & 1 & 3 & 4 & 6 \\
\hline
\end{tabular}

Table 2: Distribution of the HV flow pattern according to progression of fatty infiltration of the liver in rats.

\begin{tabular}{|l|l|l|l|l|l|l|l|}
\hline Grade & $\boldsymbol{N}$ & $\begin{array}{l}\text { hyperechogenicity } \\
\text { comparing with } \\
\text { right kidney and } \\
\text { falciform fat }\end{array}$ & $\begin{array}{l}\text { attenuation } \\
\text { of the sound } \\
\text { beam }\end{array}$ & $\begin{array}{l}\text { visibility } \\
\text { of } \\
\text { hepatic } \\
\text { vessels }\end{array}$ & \multicolumn{4}{|l}{$\begin{array}{l}\text { Histogram } \\
\text { LMEAN SD } \\
\text { NMOST/NALL }\end{array}$} \\
\hline Normal & 10 & - & - & ++++ & 19.57 & 4.73 & 34.09 \\
\hline grade I & 06 & + & - & +++ & 64.47 & 7.97 & 34.87 \\
\hline grade II & 05 & ++ & + & ++ & 70.20 & 9.11 & 31.83 \\
\hline grade III & 04 & +++ & ++ & - & 97.24 & 11.04 & 27.70 \\
\hline
\end{tabular}

Table 3: Classification of grade of fatty infiltration of the liver (B-mode) and results of gray level histograms of the dogs. $n=$ sample.

\begin{tabular}{|c|c|c|c|c|c|}
\hline Grade & $\begin{array}{c}\text { hyperechogenicity } \\
\text { comparing with } \\
\text { right kidney }\end{array}$ & $\begin{array}{c}\text { attenuation } \\
\text { of the } \\
\text { sound } \\
\text { beam }\end{array}$ & $\begin{array}{c}\text { visibility } \\
\text { of hepatic } \\
\text { vessels }\end{array}$ & $\begin{array}{c}\text { Necropsy } \\
\text { Macroscopy }\end{array}$ & $\begin{array}{c}\text { Necropsy } \\
\text { Histology }\end{array}$ \\
\hline Normal & - & - & ++++ & $\begin{array}{c}\text { smooth } \\
\text { surface, } \\
\text { brownish-red } \\
\text { color, friable } \\
\text { consistency }\end{array}$ & $\begin{array}{l}\text { Normal liver } \\
\text { parenchimal } \\
\text { architecture }\end{array}$ \\
\hline Grade I & ++ & - & ++ & $\begin{array}{c}\text { wrinkled } \\
\text { surface, } \\
\text { yellowish } \\
\text { color, friable } \\
\text { consistency }\end{array}$ & mild hepatic \\
steatosis \\
\hline Grade
\end{tabular}

Table 4: Classification of grade of fatty infiltration of the liver (B-mode) and results of necropsy of the rats. 

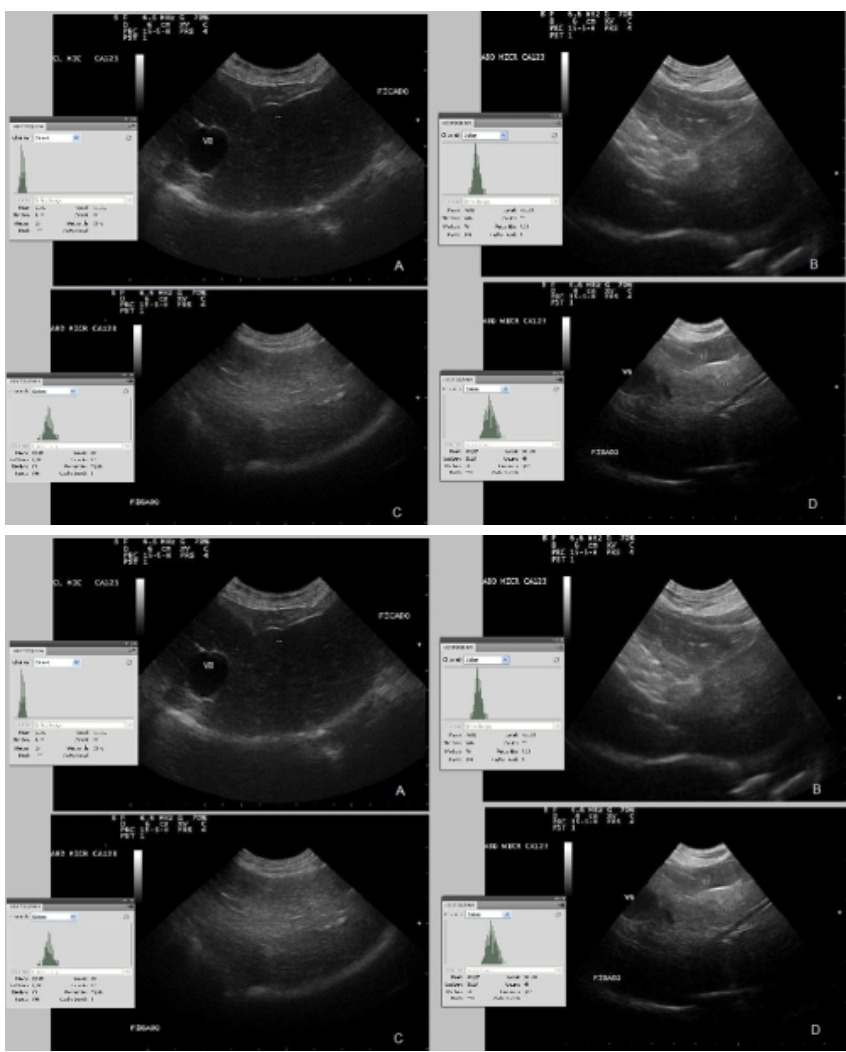

Figure 2: (A - D) Transverse B-mode sonograms of livers in dogs and respective histograms. A) Normal liver. (B) Liver with mild fatty infiltration (C) Liver with moderate fatty infiltration. (D) Liver with severe fatty infiltration. Note: as the echogenicity increases, the LMEAN values also increase.

the gold-standard noninvasive technique for such measurements in human medicine. Besides the cost of the procedure, this method has some limitations in small animal medicine because it requires multiple breath-holds and the reconstruction algorithm requires an accurate model of the fat spectra [11].

B-mode ultrasound imaging is a sensitive method to detect changes in the acoustic properties of tissues, and abnormalities are diagnosed on the basis of the recognition of changes in tissue echogenicity and texture. However, it is difficult using sonography to determine severity of diffuse liver disease. Several studies have addressed the issue of a quantitative ultrasound examination of the texture analysis of B-mode images, and in routine clinical practice, a comparison of the echogenicity of two tissues is useful [3]. Our study proposed a scale of fatty liver infiltration grade based on known sonographic criteria, as described in the Methods section. We found that at a higher grade of fatty infiltration of the liver, higher LMEAN results were obtained. These criteria for B-mode images were in accordance with the quantitative assessment of echogenicity.

In theory, the right HV seems to be the best location for the sample

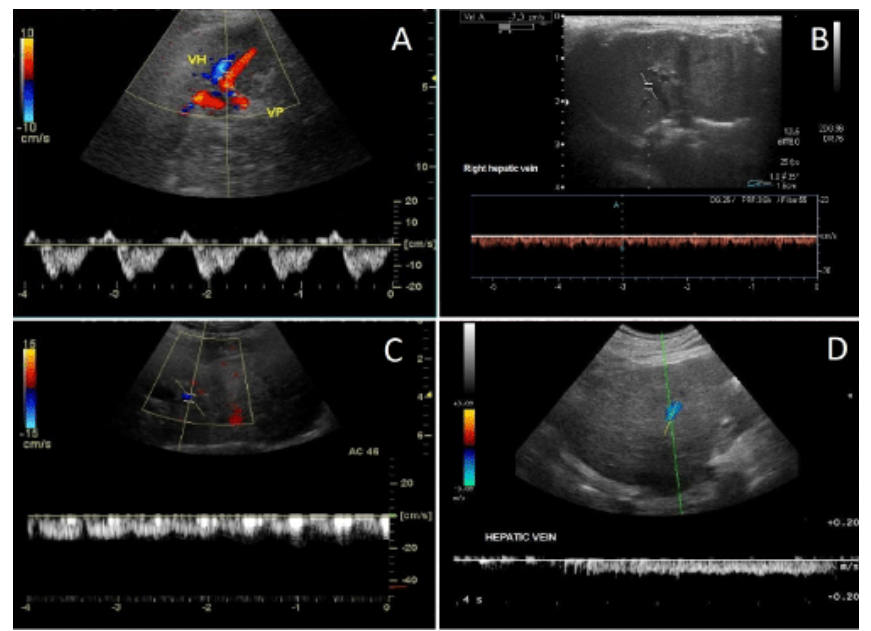

Figure 3: (A) Normal spectral Doppler of the right hepatic vein $(\mathrm{VH})$ showing a triphasic waveform in a normal dog. (VP = portal vein). (B) Spectral Doppler of the right hepatic vein in an obese rodent model with moderate to severe fatty liver disease after 8 weeks consuming the diet to induce fatty liver disease. The flow spectrum is biphasic with decreased phasic oscillation; velocity of $7 \mathrm{~cm} / \mathrm{s}$, and the angle of the sample volume correction is $35^{\circ}$. (C) Spectral Doppler of the right hepatic vein in an obese dog with moderate fatty liver disease (grade 2). The flow spectrum is biphasic without decreased phasic oscillation. (D) Spectral Doppler of the right hepatic vein in an obese dog with severe fatty liver disease (grade 3 ). The flow spectrum is abnormally monophasic.

volume of Doppler because the pulsatility of this vein would be more affected by cardiac motion than other HVs. In our study, the most common waveform of the right $\mathrm{HV}$ was triphasic in both control groups, as reported previously [16].

Obesity has become a problem in small animals, and fatty liver disease commonly occurs in obese patients and requires attention from veterinarians. We must address this disease and several differential diagnoses in routine veterinary medicine. As a result, in our study, we found abnormal HV Doppler waveforms (biphasic and monophasic) in obese animals with diffuse fatty liver infiltration. Obese dogs with moderate and severe fatty infiltration of the liver presented abnormal right HV Doppler waveforms, and pulsatility was significantly dampened, which was correlated with the severity grade of hepatic steatosis. This finding may be explained by the compressive effect of fat deposition in the hepatocytes in the HV $[17,23]$. We believe that similar to humans and rats, when hepatocytes become larger, the sinusoidal flow resistance is increased, leading to waveform changes during Doppler spectral mapping. Cardiac diseases were excluded in all dogs submitted to sonographic examination. But, obesity is known to be associated with many other interfering characteristics of the organism, such as intra-abdominal pressure, cardiac output besides intravascular filling which also may have a role to play.

In the human medical literature, is has been shown that dietary restrictions and exercise therapy improve liver histology and could

\begin{tabular}{|c|c|c|c|c|c|c|c|c|}
\hline \multirow[b]{2}{*}{ Grade of fatty liver } & \multicolumn{4}{|c|}{ Obese dogs } & \multicolumn{4}{|c|}{ Normal dogs } \\
\hline & n (15) & HV monophasic & HV biphasic & HV triphasic & n (10) & HV monophasic & HV biphasic & HV triphasic \\
\hline 0 & - & - & - & - & 10 & - & 3 & 7 \\
\hline 1 & 6 & - & 1 & 5 & - & - & - & - \\
\hline II & 5 & - & 4 & 1 & - & - & - & - \\
\hline III & 4 & 4 & - & - & - & - & - & - \\
\hline
\end{tabular}

Table 5: Distribution of sonographic grades of fatty liver infiltration and Doppler waveform of hepatic (HV) in normal and obese dogs. 
help to prevent the progression of hepatosteatosis [25]. In the present study, however, only some dogs underwent a weight loss program, and we have no data regarding whether the dampened waveform in the HV returned to a normal pattern after treatment.

One limitation of this study is the lack of information on the correlation between the ultrasound findings and the liver histology results of normal dogs. Biopsies were not performed on all dogs in this study because the dogs were non-sedated, client-owned animals. Further studies are required to determine whether an increase in echogenicity was accompanied by a gradual change of HV Doppler waveforms in spontaneous fatty infiltration of the liver also in dogs.

In conclusion, this study in rats showed that an induced gradual increase of fatty infiltration of the liver was accompanied by a gradual change of HV Doppler waveforms that is associated with the degree of fatty infiltration. HV waveforms evaluation is technically less complex than US grey scale analysis, less invasive than histologic examination of liver biopsies and finally, less expensive than magnetic resonance imaging. This assay could be helpful in diagnosing fatty infiltration of the liver and its effects on liver haemodynamics in evaluating the natural course of the disease in rats and dogs. But a correlation of these findings with the degree of fatty infiltration on histopathology remains to be determined.

Further studies are required to determine whether the HV Doppler waveform pattern could return to normal after weight loss and could be used to monitor clinical treatment.

\section{Acknowledgements}

The authors thank Flavio Cesar Viani and Raquel Sartor for their technical assistance.

The authors also thank FAPESP (Fundação de Amparo a Pesquisa do Estado de São Paulo) for the financial support of this study. Process number 2011/077083.

This manuscript represents a portion of post-doctoral research submitted by Dr. Carvalho to the Radiology Institute of University of São Paulo in partial satisfaction of the requirements for a Post-Doctoral degree.

\section{References}

1. Burkholder WJ, TOLL PW (2000) Small animal clinical nutrition. (4thed), Topeka KS: Mark Morris Institute.

2. McGreevy PD, Thomson PC, Pride C, Fawcett A, Grassi T, et al. (2005) Prevalence of obesity in dogs examined by Australian veterinary practices and the risk factors involved. Vet Rec 156: 695-702.

3. Nicoll RG, O'Brien RT, Jackson MW (1998) Qualitative ultrasonography of the liver in obese cats. Vet Radiol Ultrasound 39: 47-50.

4. Tsunoda M, Kobayashi N, Ide T, Utsumi M, Nagasawa M, et al. (2008) A novel PPARalpha agonist ameliorates insulin resistance in dogs fed a high-fat diet. Am J Physiol Endocrinol Metab 294: E833-840.

5. Kim SP, Woolcott OO, Hsu IR, Stefanoski D, Harrison LN, et al. (2012) CB(1) antagonism restores hepatic insulin sensitivity without normalization of adiposity in diet-induced obese dogs. Am J Physiol Endocrinol Metab 302: E1261-1268.

6. Wang KY, Panciera DL, Al-Rukibat RK, Radi ZA (2004) Accuracy of ultrasoundguided fine-needle aspiration of the liver and cytologic findings in dogs and cats: 97 cases (1990-2000). J Am Vet Med Assoc 224: 75-78.
7. Xenoulis PG, Steiner JM (2010) Lipid metabolism and hyperlipidemia in dogs Vet J 183: 12-21.

8. O’Brien RT, Zagzebski JA, Lu ZF, Steinberg H (1996) Measurement of acoustic backscatter and attenuation in the liver of dogs with experimentally induced steroid hepatopathy. Am J Vet Res 57: 1690-1694.

9. Syakalima M, Takiguchi M, Yasuda J, Morita Y, Hashimoto A (1998) Comparison of attenuation and liver-kidney contrast of liver ultrasonographs with histology and biochemistry in dogs with experimentally induced steroid hepatopathy. Vet Q 20: 18-22.

10. Nyland TG, Park RD (1983) Hepatic ultrasonography in the dog. Vet Radio Ultrasound 24:74-84

11. Hu HH, Kim HW, Nayak KS, Goran MI (2010) Comparison of fat-water MRI and single-voxel MRS in the assessment of hepatic and pancreatic fat fractions in humans. Obesity (Silver Spring) 18: 841-847.

12. Nyland TG, Mattoon JS (2002) Small Animal Diagnostic Ultrasound. (2nded), W. B. Saunders Company, Philadelphia, London, 93-127.

13. Carlisle CH, Jian-Xin WU, Heath TJ (1995) Anatomy of portal and hepatic veins of the dog: a basis for systematic evaluation of the liver by ultrasonography. Vet Radiol Ultrasound 36:227-33.

14. Finn-Bodner ST, Hudson JA (1998) Abdominal vascular sonography. Vet Clin North Am Small Anim Pract 28: 887-942.

15. Spaulding KA (1997) A review of sonographic identification of abdominal blood vessels and juxtavascular organs. Vet Radiol Ultrasound 38: 4-23.

16. Szatmári V, Sótonyi $P$, Vörös K (2001) Normal duplex Doppler waveforms of major abdominal blood vessels in dogs: a review. Vet Radiol Ultrasound 42 : 93-107.

17. Uzun H, Yazici B, Erdogmus B, Kocabay K, Buyukkaya R, et al. (2009) Doppler waveforms of the hepatic veins in children with diffuse fatty infiltration of the liver. Eur J Radiol 71: 552-556.

18. Nelson NC, Drost WT, Lerche P, Bonagura JD (2010) Noninvasive estimation of central venous pressure in anesthetized dogs by measurement of hepatic venous blood flow velocity and abdominal venous diameter. Vet Radiol Ultrasound 51: 313-323.

19. Smithenson BT, Mattoon JS, Bonagura JD, Abrahamsen EJ, Drost WT (2004) Pulsed-wave Doppler ultrasonographic evaluation of hepatic veins during variable hemodynamic states in healthy anesthetized dogs. Am J Vet Res 65: 734-740.

20. de Lima VM, Oliveira CP, Alves VA, Chammas MC, Oliveira EP, et al. (2008) A rodent model of NASH with cirrhosis, oval cell proliferation and hepatocellula carcinoma. J Hepatol 49: 1055-1061.

21. Szatmári V, Rothuizen J, van den Ingh TS, van Sluijs F, Voorhout G (2004) Ultrasonographic findings in dogs with hyperammonemia: 90 cases $(2000-$ 2002). J Am Vet Med Assoc 224: 717-727.

22. Kleiner DE, Brunt EM, Van Natta M, Behling C, Contos MJ, et al. (2005) Design and validation of a histological scoring system for nonalcoholic fatty liver disease. Hepatology 41: 1313-1321.

23. Rothuizen J, Desmet VJ, Van der Ingh T, Twidt DC, Bunch SE, et al. (2006) WSAVA Standards for clinical and histological diagnosis of canine and feline liver diseases. Elsevier: Philadelphia, 5-14.

24. Penninck D, D’Anjou MA (2008) Atlas of Small Animal Ultrasonography (1rsted), lowa: Balckwell Publishing, 217-280.

25. Ueno T, Sugawara H, Sujaku K, Hashimoto O, Tsuji R, et al. (1997) Therapeutic effects of restricted diet and exercise in obese patients with fatty liver. $\mathrm{J}$ Hepato 27: 103-107. 